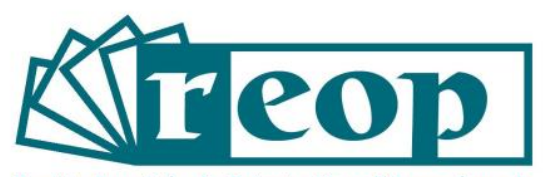

Revista Española de Orientación y Psicopedagogía

\title{
PARTICIPACIÓN DE LAS FAMILIAS, ACCIÓN TUTORIAL Y ORIENTACIÓN DESDE LA JUSTICIA SOCIAL
}

\section{PARTICIPATION OF FAMILIES, TUTORIAL ACTION AND GUIDANCE FROM A SOCIAL JUSTICE APPROACH}

\author{
Juan Antonio Bellido Cala ${ }^{1}$ \\ Universidad Nacional de Educación a Distancia, UNED. Facultad de Educación. Doctorando \\ EIDUNED. Madrid, España
}

\begin{abstract}
RESUMEN
La participación o representación constituye una de las dimensiones fundamentales que conforman, junto con la redistribución y el reconocimiento, el concepto de justicia social, cuyo significado se ha expandido durante las últimas décadas gracias a múltiples aportaciones teóricas. En el marco de nuestra investigación, hemos analizado las pautas de colaboración y participación de las familias en los centros docentes, así como la acción tutorial y orientación educativa, al entender que ambos aspectos pueden contribuir positivamente a una mejora de dicha participación y al logro de una justicia social en educación. Reviste especial interés profundizar en el análisis crítico del discurso de los docentes, como forma indirecta de indagar en la praxis educativa, así como las variaciones y divergencias que aparecen entre lo normativo y lo factual. El objetivo básico de este trabajo se centra en identificar si la justicia social, desde la dimensión participativa, está presente en la práctica educativa y el discurso de los profesionales. Metodológicamente, se parte de un enfoque cualitativo que utiliza la Teoría Fundamentada como herramienta de análisis. Los datos han sido recogidos a partir de una muestra de profesionales de la enseñanza, incluyendo orientadores, utilizando la entrevista en profundidad. Los resultados muestran que la participación no se desarrolla en términos sustantivos, sino meramente formales, evidenciando las carencias existentes en el ámbito estudiado. Las conclusiones sugieren la necesidad de una mayor implicación del profesorado en el ámbito de la atención tutorial, desarrollando técnicas y dinámicas más participativas e inclusivas. Se concluyen líneas
\end{abstract}

${ }^{1}$ Correspondencia: Juan Antonio Bellido Cala. Correo-e: jabellido@cadiz.uned.es 
esperanzadoras donde el profesorado y los y las profesionales de la orientación pueden jugar un papel esencial en la mejora de las condiciones actuales.

Palabras clave: Orientación, participación, acción tutorial, justicia social, Teoría Fundamentada.

\begin{abstract}
Participation or representation constitutes one of the fundamental dimensions that make up, together with redistribution and recognition, the concept of social justice, whose meaning has expanded during the last decades thanks to multiple theoretical contributions. Within the framework of our research, we have analyzed the patterns of collaboration and participation of families in schools, as well as the tutorial action and educational guidance, understanding that both aspects can contribute positively to an improvement of said participation and the achievement of social justice in education. It is particularly interesting to study in depth the critical analysis of teachers' discourse, as an indirect way of researching educational praxis, as well as the variations and divergences that appear between the normative and the factual. The basic objective of this work is to identify whether social justice, from the participatory dimension, is present in the educational practice and discourse of professionals. Methodologically, it is based on a qualitative approach that uses Grounded Theory as an analysis tool. Data has been collected from a sample of teachers and counselors, using in-depth interviews. The results show that participation does not take place meaningfully, but merely in a formal way, evidencing the gaps in the field studied. The conclusions suggest the need for greater involvement of teachers in the field of tutorial action, developing more participatory and inclusive techniques and dynamics. Some lines of action are suggested, in which teachers and educational counselors can play an essential role in improving current conditions.
\end{abstract}

Key Words: Guidance, participation, tutorial action, social justice, Grounded Theory.

\title{
Cómo citar este artículo:
}

Bellido-Cala, J.A. (2021). Participación de las familias, acción tutorial y orientación desde la justicia social. Revista Española de Orientación y Psicopedagogía, 32(1), 76-91. https://doi.org/10.5944/reop.vol.32.num.1.2021.30741

\section{Introducción}

La educación obligatoria deviene esencial para sentar las bases cognitivas, actitudinales y de formación de hábitos necesarios para que cualquier persona pueda seguir formándose y aprendiendo a lo largo de toda su vida, pudiendo alcanzar un nivel de vida óptimo con independencia de sus circunstancias de origen. La acción tutorial y la orientación son fundamentales para contribuir al desarrollo integral del alumnado, complementando el trabajo del 
profesorado y de la familia, así como para fomentar una educación inclusiva de calidad, en la que niñas y niños aprendan de manera óptima y se desarrollen plenamente.

Las circunstancias reales que nos rodean y en las que se vive la educación obligatoria, que pretende ser comprehensiva, nos alejan mucho en estos momentos de alcanzar con éxito la desiderata expresada en la Agenda $2030^{2}$ sobre educación inclusiva. La investigación sobre justicia social en educación que hemos desarrollado proyecta más sombras que luces, bosquejando un futuro complejo y muy alejado de los aspectos referidos en el ODS número cuatro. A través del análisis crítico del discurso de los y las profesionales de la educación y de las conclusiones preliminares obtenidas se desprenden escenarios actuales que difícilmente cohonestan con la consecución de dicho objetivo. Utilizando una aproximación cualitativa, hemos intentado identificar la presencia de la justicia social en la práctica educativa y el discurso de los y las docentes. El análisis de los relatos discursivos revela importantes desajustes entre "lo que se hace", "lo que dicen que hacen" y "lo que deberían hacer", pudiendo explicar esas diferencias el ingente corpus normativo que regula el sistema educativo, aspectos consuetudinarios como la costumbre y elementos profundamente imbricados en la vida de los centros que constituyen la cultura profesional de los docentes.

Cuenca (2012), cuando aborda la justicia social, reconoce que se trata de una noción compleja y plural, susceptible de un abordaje múltiple y mucho más subjetiva de lo que se pensaba. Nussbaum (1995, 2009), al analizar el concepto de justicia, critica las teorías que simplifican el comportamiento humano, argumentando que las explicaciones economicistas que emanan del modelo utilitarista establecen que un solo elemento puede utilizarse para medir el bienestar de los seres humanos, concluyendo que la felicidad y la justicia pueden ser acotadas y mensurables, infravalorando la complejidad de la vida humana y de las múltiples circunstancias que pueden confluir para conformar la misma.

Por su parte, Nancy Fraser (2010), considera tres dimensiones como elementos esenciales que contribuyen a la construcción del concepto de justicia social, a saber, la redistribución, el reconocimiento y la representación. Subraya que las condiciones institucionales son claves para determinar el desarrollo de prácticas justas o injustas. Descendiendo y profundizando en cada una de las dimensiones aludidas, la redistribución hace alusión al ámbito económico; la injusticia reside aquí en la inexistencia, insuficiencia o negación de los recursos necesarios para interactuar en sociedad. Respecto al reconocimiento, la desigualdad de estatus que se produce como resultado del mantenimiento de las jerarquías que se han reproducido e institucionalizado genera un problema de reconocimiento cultural. En la representación, que abunda en el aspecto político, se cristaliza la injusticia al negar la capacidad de expresión a ciertas colectividades.

Siguiendo a López-Verdugo et al. (2004), la relación que se establece entre la familia y la escuela adquiere una naturaleza esencial en la actualidad, ya que ambas instituciones se configuran como los contextos de socialización y educación más importantes para el desarrollo de los niños y niñas, asumiendo funciones complementarias e interconectadas. Como indica Epstein (2001), citada por Llevot y Bernat (2015), es muy importante el hecho de que las familias participen significativamente en el proceso educativo de sus hijos $y$, consecuentemente, establezcan unas relaciones armónicas con los centros escolares que permitan interconectar ambos espacios, el familiar y el escolar, de la mejor manera posible.

En este escenario educativo ampliado, siguiendo a Bolívar (2006, 2013), cada vez es más evidente que la institución escolar carece de la capacidad de agencia suficiente para cubrir la totalidad de las necesidades formativas de la ciudadanía. En la sociedad contemporánea de la información son muchos los agentes de socialización que compiten entre sí y entrecruzan sus influencias en la educación, instrucción y enseñanza de las nuevas generaciones. Por tanto,

\footnotetext{
2 Agenda 2030. Aprobada por la Asamblea General de la ONU, el 25 de septiembre de 2015 . Recuperado de: https://www.un.org/sustainabledevelopment/es/2015/09/la-asamblea-general-adopta-la-agenda-2030-para-el-desarrollosostenible/
} 
imputar toda la responsabilidad del resultado final, en uno $u$ otro sentido, a los centros educativos nos conduce a un camino de difícil retorno que solo redundará en un aumento del malestar, sentimiento de culpabilidad e insatisfacción de los profesionales, las familias y la ciudadanía.

Llevot y Bernad (2015) y García-Bacete (2003) indican que la participación de las familias en el centro educativo no solo aporta beneficios unidireccionales, sino que, de la misma, se nutren también los docentes y el centro, generando un considerable aumento del capital cultural y social. Se caracteriza el capital social por su peso en el mercado, valga la expresión simbólico-cultural (Bourdieu, 2008). La participación de las familias no debería limitarse a recibir información, sino que, de ser activa, supondría un plus de intervención y voluntad que redundaría en el incremento del sentido de pertenencia grupal (Pindado, 2000). García-Albadalejo y Sánchez-Liarte (2006), así como Giménez (2002), apuestan por un modelo de participación que implique la colaboración activa y permita compartir entre todos los miembros de la comunidad educativa una serie de metas y objetivos comunes para que la toma de decisiones se convierta en un proceso compartido.

En el marco de nuestro actual sistema educativo, las disposiciones legales que se han publicado y puesto en vigor han aportado un tejido normativo que ha pretendido hacer efectivo el derecho a la participación. No obstante, las prácticas escolares cotidianas no evidencian un cambio sustantivo en el ejercicio de esta. Asimismo, la Ley Orgánica de Educación (LOE, 2006) defendía el concepto de ciudadanía como elemento esencial para conocer la sociedad y aprender a integrarse en ella, como sujetos de derechos y deberes. Por contra, la Ley Orgánica de Mejora de la Calidad de la Educación (LOMCE, 2013) incorpora como conceptos-clave la competitividad y la empleabilidad. Se produce un cambio de signo altamente relevante donde se materializa el tránsito entre la formación de los ciudadanos y ciudadanas a la de personas empleadas y emprendedoras. Podría desprenderse de este cambio que el sistema educativo no preparó suficientemente a las personas para que pudieran incorporarse al mercado de trabajo y que, con la nueva norma, sí se va a conseguir ese objetivo. Lo que sí podemos apreciar es que el concepto de participación crítica y democrática queda desplazado por la emergencia de un nuevo concepto de ciudadanía, basado en parámetros productivos y del capital (Subirats, 2014)

La participación de las familias en el ámbito educativo se configura como un elemento esencial que, basándose en la equidad, posibilita los cambios y transformaciones necesarias para el logro de una ciudadanía sustantiva y plena. El concepto de participación escolar ha sido abordado con frecuencia en el ámbito de la investigación educativa. El mismo se asocia con la participación ciudadana, ya que se espera de la institución escolar que los y las estudiantes puedan desarrollar en dicho ámbito un ejercicio satisfactorio de la convivencia que les permita prepararse para la vida adulta. La implicación activa de todos los agentes educativos en el desarrollo de procesos escolares democráticos e inclusivos, así como en lo que respecta a un ethos escolar respetuoso con la diferencia y el ejercicio de los derechos de todos y todas permitiría predecir razonablemente un ejercicio pleno de la ciudadanía en la vida adulta (Ascorra et al., 2016). La participación que no trasciende los meros aspectos formales no genera cambios reales y las supuestas transformaciones pueden llegar a convertirse en mecanismos legitimadores de un orden social injusto e impuesto por los sectores sociales que detentan situaciones prevalentes de poder.

En este contexto, entendemos que la orientación educativa debe jugar un papel primordial contribuyendo a la promoción de relaciones sanas y positivas entre la familia y la escuela. La existencia de estos profesionales, con presencia en los centros docentes a través de equipos externos (EOE - Equipos de Orientación Educativa) y DO (Departamentos de Orientación, en Institutos de Educación Secundaria), y su perspectiva de trabajo global, al tratar simultáneamente con muchos agentes educativos, les confiere un rol esencial que debe ser puesto en valor y aprovechado para la mejora de las condiciones educativas existentes en los centros docentes. Además, su intervención, siguiendo a Cano y Casado (2015), debe desarrollarse en todas las etapas y con un despliegue amplio, extenso y sistemático. 
La pregunta de investigación que hemos considerado en el presente estudio es la siguiente: ¿Cómo es percibida la participación de las familias en el ámbito escolar, desde la perspectiva de los profesionales de la docencia y la orientación?

\section{Método}

Planteamos una investigación cualitativa, sustentada en el enfoque metodológico de la Teoría Fundamentada (TF) (Corbin y Strauss, 2008). Asentada en el interaccionismo simbólico, está basada en la interpretación de los escenarios sociales y en la interacción simbólica que se produce entre los sujetos (Glaser y Strauss, 2012). El propósito principal de la teoría fundamentada en los datos consiste en el descubrimiento y generación de modelos explicativos sobre un fenómeno social, partiendo del análisis sistemático y ulterior interpretación de los datos recogidos.

De acuerdo con Glaser (1992), el diseño cualitativo, a diferencia del cuantitativo, no queda conformado definitivamente al inicio de la investigación. Por el contrario, va emergiendo de manera paulatina al proceso de recogida de datos, de ahí que se caracterice por su apertura, flexibilidad y carácter netamente emergente. En estos casos, el diseño debe aspirar a conseguir, según Valles (1997), la fiabilidad, credibilidad y transferibilidad.

Para la selección de los participantes se ha realizado un muestreo intencional o discrecional, teniendo en cuenta los siguientes criterios generales: heterogeneidad, accesibilidad, disponibilidad, representatividad y tamaño. Los docentes y orientadores que han participado en el estudio desarrollan su trabajo en colegios e institutos de la provincia de Cádiz, España, tenían al menos cinco años de experiencia docente u orientadora al iniciar este estudio y el potencial de aportar información relevante de cara a los propósitos de la investigación, habida cuenta de su experiencia y consideración positiva del desempeño profesional. Un total de 32 docentes cumplieron los criterios de inclusión y decidieron participar en el estudio. De ellos fueron elegidos directamente por el investigador 12, mientras que el resto, 20, fueron seleccionados por personal de los centros educativos donde hemos trabajado. Todos y cada uno de los entrevistados fueron contactados previamente para informarles detenidamente, recabar expresamente su consentimiento y aclarar cuantas dudas plantearon sobre el marco de la investigación y el producto final de sus declaraciones. Se ha asegurado el anonimato, privacidad y confidencialidad de todos los participantes, recordándoles que su participación tenía carácter voluntario.

La técnica principal de recogida de datos ha sido la entrevista semiestructurada (Gauchi, 2017). Se han realizado entrevistas en profundidad, donde se plantearon preguntas abiertas con objeto de investigar tanto la participación de las familias en la vida de los centros, como la opinión y actitud del profesorado al respecto.

Las entrevistas se han realizado de manera individual, con una duración comprendida entre 45 y 70 minutos. Una vez establecido el clima inicial de confianza, rapport, se han realizado preguntas más detalladas y específicas con relación a la participación de las familias en el ámbito escolar, tales como: 1) ¿Cómo crees que participan las familias en el colegio? 2) ¿Podrías valorar el grado de implicación de las familias con el colegio, en cuanto a la educación de sus hijos/as? 3) ¿En tu opinión, ¿cómo deberían participar y relacionarse las familias con el centro?, entre otras muchas. 
Tabla 1

Características de la muestra.

\begin{tabular}{|c|c|c|c|c|c|c|c|c|}
\hline $\begin{array}{c}\text { Etapa } \\
\text { Educativa }\end{array}$ & $\begin{array}{c}\text { Hombres } \\
\mathbf{H}\end{array}$ & $\begin{array}{c}\text { Mujeres } \\
\text { M }\end{array}$ & $\begin{array}{c}\text { Edad } \\
\mathbf{H}\end{array}$ & $\begin{array}{c}\text { Edad } \\
\text { M }\end{array}$ & $\begin{array}{c}\text { Media de } \\
\text { edad } \\
\text { H }\end{array}$ & $\begin{array}{l}\text { Media de } \\
\text { edad } \\
\text { M }\end{array}$ & Ciudad $_{1}$ & Total \\
\hline $\begin{array}{l}\text { IES } \\
\text { Secundaria }\end{array}$ & 6 & 6 & [44-69] & [41-56] & 55.8 & 47.2 & $\begin{array}{l}\text { A: } 1 \\
\text { B: } 1 \\
\text { C: } 10\end{array}$ & 12 \\
\hline $\begin{array}{l}\text { Infantil y } \\
\text { Primaria }\end{array}$ & 5 & 9 & [45-62] & [34-61] & 57 & 48 & $\begin{array}{l}\text { A: } 6 \\
\text { B: } 2 \\
\text { C: } 6\end{array}$ & 14 \\
\hline $\begin{array}{l}\text { Equipos } \\
\text { Directivos }_{2}\end{array}$ & $11_{2}$ & $10_{2}$ & [40-69] & [45-61] & 55.8 & 51.9 & $\begin{array}{l}\text { A: } 4_{2} \\
\text { B: } 3_{2} \\
\text { C: } 14_{2}\end{array}$ & $21_{2}$ \\
\hline Otros $_{3}:$ & 4 & 2 & [40-64] & [37-43] & 52.5 & 58.5 & $\begin{array}{l}\text { A: } 0 \\
\text { B: } 1 \\
\text { C: } 5\end{array}$ & 6 \\
\hline TOTAL & 15 & 17 & [40-69] & [37-61] & 55.3 & 46.8 & $\begin{array}{l}\text { A:7 } \\
\text { B: } 4 \\
C: 21\end{array}$ & 32 \\
\hline
\end{tabular}

${ }_{1}$ Ciudades dentro de la provincia de Cádiz (España), donde A: Jerez de la Frontera; B: Cádiz; C: La Línea de la Concepción. ${ }_{2}$ Contabilizado ya en sectores del profesorado (IES y Maestros/as). Ejercen o han ejercido funciones directivas en los centros docentes. ${ }_{3}$ Asesores de formación, Inspección y Orientadores Externos (EOE).

Fuente: Elaboración propia.

Se analizó la información recogida utilizando un procedimiento denominado análisis comparativo constante, compuesto de tres fases de codificación distintas y consecutivas: abierta, axial y selectiva. Tanto la recogida como el análisis de los datos del estudio se produjo de manera simultánea, surgiendo la teoría a partir de dicho proceso. El análisis de los datos prosiguió, desde la primera entrevista hasta que se alcanzó el nivel de saturación (Glaser y Strauss, 1967), también denominado punto de redundancia (Lincoln y Guba, 1985), todo ello con objeto de garantizar los criterios éticos y de rigor científico de la investigación cualitativa llevada a cabo (Noreña et al., 2012).

Se cifró y codificó el texto transcrito y se refinó la codificación a medida que nuevos temas emergentes fueron surgiendo. Asimismo, se procedió a categorizar los códigos clasificados, comparándolos e interpretándolos en el marco de las transcripciones generales.

El método en la TF tiene carácter circular, dado que permite al investigador analizar los datos obtenidos, comenzando inmediatamente su codificación y categorización. Representa, asimismo, un escenario metodológico flexible, ya que permite modificar el foco de atención inicial y ahondar en otra dirección estratégica que haya podido ser puesta de manifiesto a partir de los nuevos datos recopilados y analizados. Se trata de un proceso creativo, dinámico, cíclico y reflexivo, que progresa de manera continua (Taylor y Bodgan, 1986). 


\section{Resultados}

El fenómeno estudiado, la participación de las familias en la escuela, viene generado por una serie de condiciones causales, siendo estas todos los acontecimientos, sucesos o premisas que pueden tener algún tipo de influencia sobre dicho fenómeno y lo generan. Podemos rastrear dichas condiciones si somos capaces de averiguar cómo, dónde y por qué se produce dicho fenómeno. En primer lugar, se procedió a utilizar una aproximación utilizando la codificación abierta. Siguiendo a Corbin y Strauss (2008), identificamos a partir de este procedimiento analítico tanto los conceptos como las propiedades, características y dimensiones de los datos obtenidos. Fuimos asignando los diferentes códigos que describían el fenómeno objeto de estudio de manera más extensa y provechosa para nuestros fines. Simultáneamente a la recopilación de la información codificamos los datos recabados, redactando los memorandos que evolucionaron de menor a mayor conceptualización. Asimismo, dichos memorandos evidenciaron las relaciones potenciales entre las distintas categorías (Monge, 2015). Emergieron y pudimos analizar once códigos, todos ellos relacionados entre sí pero singulares en tanto en cuanto se diferenciaban del resto de códigos: 1) actitud del profesorado hacia la participación, 2) participación de las familias como representación, 3) intereses del profesorado, 4) visión corporativista y limitada del profesorado, 5) obstáculos para la participación, 6) liderazgo y toma de decisiones, 7) iniciativa, 8) organización escolar, 9) identidad y pertenencia, 10) motivación del profesorado y, finalmente, 11) normativa de aplicación. Esta primera codificación se redujo, tras la determinación de las propiedades y dimensiones, a cinco categorías comprehensivas, que integraban todas y cada una de las anteriores, como son: 1) liderazgo, 2) organización escolar, 3) patrones de participación, 4) normativa y 5) actitud hacia la participación de las familias. Estas categorías representan, asimismo, las condiciones causales establecidas por la codificación axial. El tránsito entre la codificación abierta y axial se produjo por decantación de los once primeros códigos en otros cinco que, a nuestro entender, tenían carácter comprehensivo y aglutinante.

En la codificación selectiva, se determinó la existencia de una categoría central. El propósito de la recopilación selectiva era saturar conceptualmente las categorías, como paso previo al estudio de la literatura existente al respecto (Monge, 2015). Hemos tenido en cuenta los vínculos e interacciones con el resto de las categorías y procurado que todas y cada una de ellas pudieran estar relacionadas con la central, quedando definida esta como "percepción de los docentes sobre la participación de las familias y organización escolar".

Trasladando la codificación explicada a una valoración cualitativa de los hallazgos, comenzaremos indicando que el profesorado asume con naturalidad la presencia de las familias en la escuela, interpretándola desde una lógica asimétrica, donde ha de prevalecer el liderazgo organizativo y personal de los docentes, ubicándoles en una situación prevalente que refuerza el estatus de superioridad del rol profesional. Las familias son aceptadas siempre y cuando desempeñen el papel subordinado de auxiliares y ayudantes, cobijadas bajo el control del profesorado y desde una relación basada en la desconfianza ya que, de otro modo, podrían llegar a convertirse en unos agentes de control del centro y los profesionales que trabajan en él.

...Hombre..., las familias sí son aceptadas, sobre todo en los niveles de Infantil porque las maestras necesitan mucho la colaboración de los padres cuando ellas..., cuando el profesorado necesita hacer algo, pero cuando necesitan pues... montar un día de Halloween, montar una zambomba, ir de excursión... para eso es para lo que utilizan a los padres... los utilizan nada más que para eso, para el apoyo logístico para montar cualquier evento o cualquier cosa, pero después cuando esos padres les piden algo al profesorado para que hagan o no... los profesores están muy, ..., no les gusta, vamos, que el padre entre en ese terreno, entonces más bien fuera. A mí los padres me sirven para ayudarme, siempre con un respeto... para que tampoco sea aquello, que me vayan a dirigir mi trabajo... $(\mathrm{E} 1)$. 
Se considera la participación familiar como buena o satisfactoria siempre y cuando las familias participen de manera puntual, asumiendo un rol secundario desde donde colaboran en labores logísticas y accesorias durante el curso escolar, como son: eventos y efemérides, excursiones y salidas extraescolares, convivencias, ornamentación y arreglos de aulas y espacios del centro o la búsqueda de recursos materiales o logísticos.

...Con los padres también hay un problema, que como se les de mucha confianza se creen..., te hacen la programación de las clases, como te descuides (risas suaves...) te programan el curso y tienes que hacer lo que ellos digan. Entonces, ante eso, pues muchas veces es mejor tenerlos un poquito... (risas...) a raya, porque... hay de todo. Hay padres respetuosos, que llegan nada más que para colaborar y lo que usted diga, y después está el padre o la madre, normalmente la madre, ummm..., que se quiere entrometer en todo.... si tú controlas a los padres, te sirven mucho de ayuda, pero tienes que tener el carácter de controlarlo... (E18).

Ese formato deficitario de participación se produce a pesar de que toda la normativa reguladora de la organización de los centros docentes aboga por la participación amplia y efectiva de padres y madres en la educación de sus hijos.

...Este tipo de procesos conlleva mucho esfuerzo y tiempo. Los padres estamos en una sociedad en la que la participación no es... digamos... una seña de identidad, salvo cuando hay un problema gordo o pico de trabajo, no como norma.... En cuanto a participación... somos una sociedad bastante individualista. La escasa participación en la escuela puede ser un reflejo de la escasa participación social. La participación es... bastante más nominal que real... (E10).

Los y las docentes aceptan y toleran la participación, aunque se produce cierto rechazo a permitir a las familias ampliar su rango de actuaciones. Se aprecia que el profesorado de Educación Secundaria y, en general, los docentes noveles presentan un mayor grado de inquietud personal a la hora de relacionarse con las familias, viviendo estas situaciones como amenazas a su incipiente estatus e identidad profesional, aún no consolidados, dado que temen una injerencia incontrolada e incontrolable en su labor docente por parte de agentes externos, las familias. Este aspecto queda en evidencia cuando se analiza la acción tutorial. Muchos profesores carecen de las herramientas necesarias para llevar a buen término la relación con el alumnado y sus familias.

Prácticamente todos los profesionales de la orientación entrevistados coinciden en este aspecto, así como en la necesidad de reforzar los lazos entre la orientación y la función tutorial. Las sinergias positivas que pueden crearse entre estos profesionales, así como de la transferencia de conocimiento experto permitirían aumentar la cualificación de los docentes y, consecuentemente, mejorar la atención a las familias. Hemos detectado que este tipo de dinámicas son más fluidas en Educación Secundaria, donde la figura del o la profesional de la orientación está permanente dentro de los Institutos de Educación Secundaria. En los Colegios de Educación Infantil y Primaria, donde dicho profesional forma parte de un equipo externo es mucho más difícil propiciar este tipo de relaciones, asignándole a la persona que ejerce la orientación un rol más clínico y diagnóstico, alejado del marco inclusivo y pedagógico que sería deseable.

...La mayoría del profesorado que llega sin experiencia (novel) tiene como actitud fundamental la queja... hay que estar recordándoles continuamente todo... esa inexperiencia nos está repercutiendo negativamente. El discurso de este profesor... culpabiliza al alumnado y a las familias, pero hay que insistirle para que hable y se relacione con las familias. Hay una minoría que esperan una solución externa, te lo dicen así... no puedo con esto... Incluso las tutorías con las familias han tenido que ser tutorizadas en algunos casos por el equipo directivo y jefatura de estudios y... parece que en algunos profesores existe temor al mero hecho de 
relacionarse con las familias. Tenemos casos de profesoras que... desprecian y humillan a los alumnos... y no te digo con respecto a las familias... (E22).

Como orientadora soy consciente de que no es fácil, al menos al principio, intentar colaborar con los tutores. Lo suelen ver... digamos... como una injerencia en su trabajo y funciones. No obstante, poco a poco he podido apreciar cambios en el Instituto a lo largo de los últimos años. Eso sí... (risas), no hay que desfallecer... porque no es fácil... (E5).

El profesorado maneja habitualmente una serie de hipótesis explicativas que intentan justificar sus roles profesionales e identitarios al tiempo que defienden el papel auxiliar y subsidiario de las familias en el ámbito escolar. Creen que se está produciendo una amplia cesión de soberanía por parte de las familias hacia la institución escolar debido, en muchos casos, a la manifiesta incapacidad familiar para abordar los rudimentos básicos de la educación de sus hijos e hijas. Se sobrecarga a la escuela con tareas ajenas a la institución educativa y eso impide a los docentes desarrollar con plenitud sus funciones esenciales, al tiempo que los somete a un continuo e insistente escrutinio por parte de los de padres y madres.

...Los padres son rehenes de la situación actual, bien por trabajo o paro... el padre está poco tiempo en casa, el alumno se encuentra solo, tiene su llave... Los padres han perdido el papel, la auctoritas que tenían... democracia sí, pero la autoridad es la autoridad. Cada vez se va perdiendo más la cultura del esfuerzo, se prima poco por parte de las familias... Asisten cuando se les llama. Participan activamente en la vida del Consejo Escolar y, sorprendentemente, tenemos incluso padres de reserva... (E7).

Los aspectos formales de la participación de las familias son respetados con rigor y sin que pueda imputarse a los centros escolares y equipos directivos negligencia o desidia en este sentido. El problema es que estos aspectos reglamentados difícilmente penetran en el tejido real del funcionamiento de las instituciones escolares y se limitan a recoger que la participación es real, efectiva y respetuosa con las leyes.

Los procesos de comunicación entre familias y escuela se articulan a través de tutorías o reuniones, delegados de clase, consejos escolares, asociaciones de padres y madres y reuniones o entrevistas para la recogida de calificaciones. En este marco relacional, el vínculo es claramente asimétrico y los padres y madres acuden al centro, en la mayoría de las ocasiones, a demanda de la escuela y tras la citación por parte del profesorado. Desarrollan las familias un papel de perceptor de servicios (clientes) que establece límites rígidos y reglamentados a la hora de relacionarse con la institución escolar.

...En líneas generales existe buena relación con las familias, cada vez hay mayor implicación, en general. Hay familias que no se implican, que tienen dejadez... Creo que es más un... parámetro social... por ejemplo, los típicos padres protectores que el niño no quiere estar aquí se dedican a molestar... y no tenemos alternativas... y cuando tú llamas al padre, en vez de apoyar las medidas para encauzar el tema, nos dice que le tenemos harto, que se le tiene manía... Las familias participan a través del AMPA, ... y luego a través de las evaluaciones y las reuniones con los tutores... (E21).

...Como orientador de un equipo externo percibo muchos recelos por parte del profesorado. Nos ven como elementos coactivos (risas), cuando nuestra principal misión es ayudarlos. Podríamos aportar mucho, a partir de nuestro conocimiento y cualificación, si nos dejaran intervenir y ayudarles. No somos solo un profesional que diagnostica trastornos del aprendizaje, pero eso es algo demasiado arraigado en la cultura de los centros que es muy difícil desterrar.... (E23). 
A partir de las entrevistas realizadas extraemos una visión muy amplia de la situación actual en la escuela. Los profesionales de la orientación, al estar obligados a relacionarse con todo el profesorado y las familias disponen de una información de carácter privilegiado que hemos valorado como de gran interés para triangular nuestro estudio. En muchos casos nos trasladan las posibilidades que se abren para mejorar la relación entre la familia y la escuela, incidiendo positivamente en la participación de aquellas en el ámbito de la institución escolar.

\section{Conclusiones y Discusión}

Los resultados del presente estudio confirman los hallazgos de otras investigaciones analizadas con relación a la participación de las familias en la institución escolar. En el marco de un estudio que investigó la influencia del apoyo y participación parental en la educación de sus hijos y la influencia de dicha actitud colaborativa en la mejora de sus logros académicos, Seng et al. (2016) concluyeron que la participación de los padres y madres en la escuela, a través de la comunicación con los docentes así como manifestando interés y colaboración en la realización de tareas y actividades, ayudando a sus hijos e hijas, es un factor altamente relevante que influencia positivamente el logro académico del alumnado. Estos investigadores han señalado que los padres deberían de invertir mucho más tiempo en la supervisión de todo lo referido a la escuela, incorporando en ese elenco de temas de interés las actividades, expectativas y demás rudimentos académicos referidos a sus hijos e hijas. En la medida en que dicha participación se manifiesta de manera sistemática y adecuada, el alumnado podría mejorar significativamente su desempeño académico en la escuela. Estamos de acuerdo, sin duda, en que este tipo de participación parental, alejado muchas veces de la rutina diaria que hemos encontrado en nuestros hallazgos, mejoraría sensiblemente todas las áreas de participación familiar en el ámbito escolar y, por tanto, reforzaría significativamente la relación entre la familia y la escuela.

En la misma línea que el estudio anterior, Assefa y Sintayehu (2019) concluyen que existe una correlación positiva entre la implicación y participación de los padres y madres y el logro académico de sus hijos e hijas, siendo esta actividad parental muy recomendada tanto para reforzar los aspectos académicos como para incrementar y reforzar el vínculo con la escuela. Dicho esto, coincidimos en nuestros hallazgos con los que recogen los autores, habida cuenta que el éxito de este tipo de intervenciones de los progenitores no alcanza a toda la población escolar, lo que redunda negativamente en los dos aspectos anteriormente referidos, el rendimiento y la vinculación con la institución. Por el contrario, el tipo de relaciones que más abundan entre las familias y los docentes, como hemos recogido también en nuestro estudio, se limita muchas veces a determinados aspectos, que, aunque importantes, son escasamente relevantes para profundizar en la relación. Dichos elementos podrían considerarse como meramente logísticos o instrumentales, tales como proveer de material escolar a sus hijos, llevarlos al colegio y hablar ocasionalmente con los maestros y profesores. Los autores concluyen que los resultados de su estudio no pueden ser extrapolados automáticamente a otros contextos, al igual que nuestra investigación, que no pretende generalizar los hallazgos obtenidos, habida cuenta de la complejidad del sistema escolar y de la diversidad de todos los agentes implicados, como son familias, profesorado y alumnado, además del contexto geográfico donde se ha realizado la investigación, con sus particulares características sociodemográficas.

También coincidimos con Armas (2012), que concluye en su estudio que existe una buena predisposición, a priori, por parte del profesorado para recibir a las familias, no percibiendo 
obstáculos para la misma. Entendemos, en nuestro caso, que las barreras a la participación no son formales ni explícitas, de ahí la complejidad de erradicarlas. Asimismo, estamos de acuerdo con la autora en que la participación de las familias está fuertemente ligada al nivel sociocultural de las mismas y a determinados aspectos identitarios que facilitan, por basarse en un sentido de pertenencia a la institución, dicha relación con la institución escolar.

Con relación a las hipótesis manejadas por el profesorado para explicar la escasa participación de las familias y, en algunos casos, el desapego, Anabalón et al. (2008) llegaron a la conclusión que la jornada laboral de las familias (padres y madres) constituía uno de los principales elementos que contribuían a explicar, dado su carácter limitante, la escasa participación de las familias en la escuela. Los hallazgos del presente estudio concuerdan con los de los autores citados.

Otra importante correspondencia la encontramos en Oraisón y Pérez (2006), donde coincidimos con las autoras al considerar que las relaciones entre la familia y escuela se plantean desde una lógica asimétrica, donde los que son poseedores de la información profesional, ubicados en una posición prevalente, la transmiten a los padres y madres sin posibilidad, la mayor parte de las veces, de cuestionarla, limitándose estos últimos a recibirla de manera pasiva e intentando ajustar su comportamiento a los requerimientos impuestos por la institución escolar. La posición activa y decisoria de la escuela contrasta dramáticamente con el rol pasivo asumido por las familias, cuya presencia en la escuela se caracteriza por ser un sector obediente, pasivo y sometido.

Siguiendo la misma línea del estudio anterior, Ceballos y Saiz (2019) concluyen, al igual que nuestros hallazgos, que las relaciones que se establecen entre los docentes y las familias prácticamente se limitan al intercambio de información, revistiendo la misma un carácter básicamente unidireccional. Dicha interacción está basada en un desequilibrio de poder, ya que las familias recurren a los profesionales de la enseñanza en calidad de expertos con objeto de recibir soluciones prefijadas para problemas muy concretos, limitando así las posibilidades de una construcción identitaria conjunta de la relación entre ambos sectores, familia y escuela.

Por su parte, Bourdieu $(2007,2008)$ reconoce, tal y como hemos encontrado en nuestro estudio, las importantes barreras y cortapisas que las instituciones escolares imponen, de manera mayoritariamente encubierta, a la participación padres y madres en la escuela, coartando el establecimiento y desarrollo de unas relaciones fluidas entre estos dos agentes, pese al impacto desfavorable que tal escenario puede suponer para la relación de niños y niñas, así como de las familias con la escuela.

La participación de los padres y madres en el ámbito escolar, según Hoover-Dempsey et al. (1995), se vería favorecida si estos son invitados a participar y perciben oportunidades reales de hacerlo. Como hemos podido comprobar a lo largo de nuestra investigación, salvo para aquellos ámbitos anecdóticos y auxiliares, la participación de las familias no es demandada en un plano más igualitario por los profesionales de la docencia. No podemos olvidar, como apuntan Leonard et al. (2013), que los profesionales de la educación, tanto profesorado como directivos, deberían promover la participación de las familias ya que este aspecto, según las investigaciones de estos autores, estimularía los procesos de identificación con la escuela y permitiría la construcción de un sentido identitario de pertenencia hacia la institución. Para ello es preciso que se haga partícipes a los padres y madres de las decisiones y no solo acogerlos o tolerarlos mientras que se comporten adecuadamente y no molesten, como se recoge en los hallazgos de nuestro estudio.

Encontramos también importantes convergencias de nuestro trabajo con la investigación de García-Cano et al. (2015), cuyos hallazgos indican que los centros educativos, incluso tras someterse a procesos de transformación escolar, no consiguen elevar las tasas de participación de aquellas familias que, como han indicado muchos de nuestros entrevistados, no participan de ningún modo en la vida de la escuela. En ese sentido, según las autoras, existía una tendencia significativa por parte del profesorado a realizar interpretaciones basadas en el déficit y la 
diferencia. Esto último ha podido ser constatado repetidamente en nuestras entrevistas, donde la carga de la responsabilidad por esa falta de colaboración es imputada básicamente a los padres y madres, evitando cualquier análisis que pudiera afectar negativamente a la identidad o autoestima profesional de los docentes.

Nuestros resultados concuerdan con las conclusiones del estudio de Etxeberria e Intxausti (2013), que indica que las familias, cuando lo hacen, se implican de manera personalizada con los tutores, existiendo un número menor de interacciones a nivel grupal.

La práctica habitual de los profesionales de la docencia discurre por derroteros que no siempre facilitan la participación de los padres y madres que exceda el cauce "natural" por el que debe discurrir la relación familia-escuela.

Se constata la existencia de un mayor nivel de participación cuando se trata de prestar apoyo instrumental a la escuela en lo concerniente al rendimiento académico de sus hijos. El profesorado destaca este aspecto como uno de los indicadores para ponderar la participación e implicación de las familias en el centro.

La investigación donde se inserta el presente estudio pretende analizar las prácticas educativas que avalen, de manera sustantiva y real, la existencia de la justicia social en educación. Los y las profesionales de la orientación manifiestan que pueden hacer mucho más de lo que les dejan hacer. Su contribución al empoderamiento del profesorado, aportándole técnicas de trabajo y asesoramiento, es algo que debiera generalizarse en la práctica de los centros.

Hemos de señalar las limitaciones que nos hemos encontrado a la hora de desarrollar este estudio y que se han centrado en las entrevistas mantenidas con el profesorado. Nos interesaba su visión, extraída a partir del análisis de su discurso. Sin la menor duda, podemos establecer como línea de proyección futura la necesidad de complementar esta investigación y ampliarla incorporando al sector de las familias para contrastar los hallazgos obtenidos y triangular las conclusiones que pudieran obtenerse más adelante.

Uno de los componentes de la tríada en la que se sustenta el concepto de justicia social, esto es, la representación o participación, no adquiere, según nuestros hallazgos, la dimensión sustantiva que sería necesaria para considerar que su presencia es plena en el ámbito de las instituciones escolares estudiadas, lo que redundaría en el perjuicio subsiguiente para uno de los sectores intervinientes, las familias, que no puede ubicarse en un plano de igualdad democrática y participativa en el seno de una institución, la escuela, que deviene esencial para la formación de los ciudadanos y sociedades contemporáneas. El papel de los orientadores y orientadores deviene esencial a la hora de prestar asesoramiento a las familias, especialmente en aquellos casos en los que se detecta un mayor distanciamiento de estas hacia la institución escolar. En este sentido, lejos de actuar como elementos aislados, la práctica y experiencia de estos profesionales evidencia que la coordinación con otros recursos, tanto internos del centro como externos a la institución escolar genera sinergias altamente positivas que redundan en beneficio no solo de las familias, sino de todos los agentes educativos y, por tanto, de la institución escolar en su conjunto.

Las instituciones educativas perseveran en la reproducción de las marcadas diferencias sociales que caracterizan a nuestra sociedad. Es necesario un cambio cultural profundo en el ámbito de las corporaciones educativas para que la toma de conciencia social real pueda permitir desarrollar modelos que tracen el camino hacia un sistema escolar más justo, respetuoso y considerado con las múltiples diferencias humanas que coexisten en él. Creemos que orientadores y orientadoras pueden aportar un conocimiento experto que permita al resto de los y las docentes potenciar al máximo su papel y contribuir, de ese modo, a la inclusión y equidad escolar, así como el ejercicio de una docencia crítica y comprometida. Hemos encontrado que la organización en red de los profesionales de la orientación, en el marco de una localidad o zona educativa, les permite desarrollar su trabajo de manera más competente. Compartir los problemas, análisis y posibles soluciones les hace crecer profesionalmente y permite, a su vez, exportar hallazgos y mejoras a 
otros centros docentes, redundando todo ello en la mejora y prestigio de su labor ante la comunidad educativa.

Creemos que el análisis del discurso del profesorado es importante para que los y las profesionales de la orientación lo tengan en cuenta y, a partir del mismo, desarrollar planes de trabajo con el profesorado que permitan actualizar y mejorar los planes de acción tutorial y las competencias de los y las docentes a este respecto. También es importante dicho análisis de cara al diseño de los planes de formación del profesorado (Martínez et al., 2000).

Finalmente, el papel del profesorado, el factor humano, una vez más, resulta absolutamente clave en este escenario, donde la participación de los sectores interesados en el ámbito educativo pueda permitir representarlos en un plano de igualdad que redunde en beneficio de todos ellos: alumnado, familias y profesorado, con el apoyo de los y las orientadores, como agentes que pueden contribuir a fomentar y enriquecer esta relación mutua.

\section{Referencias bibliográficas}

Anabalón, M., Carrasco, S., Díaz, D., Gallardo, C. y Cárcamo, H. (2008). El compromiso familiar frente al desempeño escolar de niños y niñas de educación general básica en la ciudad de Chillán. Horizontes $\quad$ Educacionales, 13(1), 11-21. https://www.redalyc.org/articulo.oa?id=97912446001

Armas, N. (2012). Percepción del profesorado sobre la participación de las familias en la escuela, especialmente de aquellas en situación de riesgo psicosocial. IPSE-ds, Revista de intervención psicosocioeducativa en la desadaptación social. IPSE-ds 2012 Vol. 5 ISSN 2013-2352 pag. 9-23. http://ipseds.ulpgc.es/w IPSE-ds05a01.pdf

Ascorra, P., López, V., y Urbina, C. (2016). Participación estudiantil en escuelas chilenas con buena y mala convivencia escolar. Revista de psicología (Santiago), 25(2), 01-18. https://scielo.conicyt.cl/scielo.php?script=sci arttext\&pid=S0719-

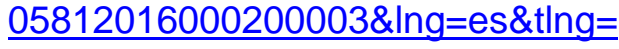

Assefa, A. y Sintayehu, B. (2019). Relationship between Parental Involvement and Students' Academic Achievement in Model Primary and Secondary School of Haramaya University, East Hararghe Zone, Oromia Regional State, Ethiopia. International Journal of Education \& Literacy Studies, 7(2), 46-56. https://eric.ed.gov/?id=EJ1219590

Bolívar, A. (2006). Familia y escuela: dos mundos llamados a trabajar en común. Revista de educación, 339, 119-146. http://www.educacionyfp.gob.es/revista-de-educacion/numerosrevista-educacion/numeros-anteriores/2006/re339/re339-07.html

Bolívar, A. (2013). Autonomía y participación en el contexto actual. Participación educativa, 2 (2), 81-88. https://sede.educacion.gob.es/publiventa/detalle.action?cod=16058

Bourdieu, P. (2007). Cosas Dichas. Gedisa.

Bourdieu, P. (2008). Capital cultural, escuela y espacio social. Siglo XXI.

Cano, R. y Casado, M. (2015). Escuela y familia. Dos pilares fundamentales para unas buenas prácticas de orientación educativa a través de las escuelas de padres. Revista Electrónica 
Interuniversitaria de Formación del Profesorado [en línea]. 2015, 18(2), 15-28. https://www.redalyc.org/articulo.oa?id=217036214003

Ceballos, N. y Saiz, A. (2019). La acción tutorial como escenario de colaboración familia-escuela. Revista Española de Orientación y Psicopedagogía, 30(2), 28 - 45. https://doi.org/10.5944/reop.vol.30.num.2.2019.25336

Corbin, J. y Strauss, A. (2008). Basics of qualitative research: Techniques and procedures for developing grounded theory. Sage.

Cuenca, R 2012. Sobre justicia social y su relación con la educación en tiempos de desigualdad, RINACE. Red Iberoamericana de Investigación sobre Cambio y Eficacia Escolar. 1(1), 2012, pp. 79-93. http://www.rinace.net/riejs/numeros/vol1-num1/art3.pdf

Epstein, J. (2001): School, family, and community partnerships: preparing educators and improving schools. Westview Press.

Etxeberria, F. y Intxausti, N. (2013). La percepción de los tutores sobre la implicación educativa de las familias inmigrantes en la comunidad autónoma del País Vasco. Revista Española de $\begin{array}{lllllll}\text { Orientación } & y & \text { Psicopedagogía, } & 24(3), & 43 & - & 62 .\end{array}$ https://doi.org/10.5944/reop.vol.24.num.3.2013.11244

Fraser, N. (2010). Scales of justice. Columbia University Press.

García-Albadalejo, A. y Sánchez-Liarte, C. (2006): La participación de los padres en los documentos institucionales de los centros educativos, en A. García Albaladejo (coord.). Participación de las familias en la vida escolar: acciones y estrategias (pp. 149-180). Ministerio de Educación y Ciencia.

García-Cano, M., Antolínez-Domínguez, I. y Márquez-Lepe, E. (2015). Del déficit a la norma: representaciones sociales sobre familias y participación escolar. Convergencia, 22(69), 181-211.http://www.scielo.org.mx/scielo.php?script=sci arttext\&pid=S1405$14352015000300181 \& \operatorname{lng}=\mathrm{es} \& \ln \mathrm{l}=\mathrm{es}$

García-Bacete, F.J. (2003). Las relaciones escuela-familia: un reto educativo. Infancia y Aprendizaje, 26 (4), 425-438. http://dx.doi.org/10.1174/021037003322553824

Gauchi, V. (2017). Estudio de los métodos de investigación y técnicas de recolección de datos utilizadas en bibliotecología y ciencia de la información. Revista Española de Documentación Científica 40(2), e175. https://doi.org/10.3989/redc.2017.2.1333

Giménez, C. (2002). Dinamización comunitaria en el ámbito de la inmigración: Apuntes y propuestas sobre participación, mediación y codesarrollo, en M. J. Rubio y S. Monteros (coords.) La exclusión social. Teoría y práctica de la intervención (pp. 99-127). Editorial CCS.

Glaser, B. (1992). Basics of grounded Theory analysis: Emergence versus forcing. Sociology Press.

Glaser, B. y Strauss, A. (1967). The discovery of Grounded Theory. Aldine.

Glaser, B. G. y Strauss, A. L. (2012). The discovery of grounded theory: strategies for qualitative research. Transaction Publisher.

Hoover-Dempsey, K. V., Bassler, O. C. y Burow, R. (1995). Parents' reported involvement in students' homework: Strategies and practices. Elementary School Journal, 95, 435-450. https://doi.org/10.1086/461854 
Leonard, H. S., Lewis, R., Freedman, A. M., Passmore, J., Lines, R. y Selart, M. (2013). Participation and Organizational Commitment during Change. From Uto-pist to Realist Perspectives. John Wiley \& Sons.

Lincoln, Y.S. y Guba, E.G. (1985). Naturalistic enquiry. Sage.

LOE. Ley Orgánica 2/2006, de 3 de mayo, de Educación. Texto publicado en BOE el 4/05/2006.

LOMCE. Ley Orgánica 8/2013, de Mejora de la Calidad de la Educación. Texto publicado en BOE, el 10/12/2013.

López-Verdugo, I., Ridao-Ramírez, P. y Sánchez-Hidalgo, J. (2004). Las familias y las escuelas: una reflexión acerca de entornos educativos compartidos. Revista de Educación, 334, 143163.http://www.educacionyfp.gob.es/dam/jcr:519936b7-c72b-4905-bfb1994b2c1c3925/re33410-pdf.pdf

Llevot, N. y Bernat, O. (2015). La participación de las familias en la escuela: factores clave. Revista de la Asociación de Sociología de la Educación I rase.ase.es I ISSN 1988-7302 I 8(1), 57-70. https://ojs.uv.es/index.php/RASE/article/view/8761

Martínez-González, R.A., Pereira, M., Rodríguez, B., Peña, A., Martínez, R., García González, M.P., Donaire, B., Álvarez, A.I. y Casielles, B. (2000). Dinamización de las relaciones familia-centro escolar a través de la formación del profesorado en este campo de actuación. Revista Española de Orientación y Psicopedagogía, 11(19), 107-120. http://doi.org/10.5944/reop.vol.11.num.19.2000.11326

Monge, V. (2015). La codificación en el método de investigación de la grounded theory o teoría fundamentada. Innovaciones educativas, 17, 22, 77-84. https://doi.org/10.22458/ie.v17i22.1100

Noreña, A., Alcaraz, N., Rojas, J. y Rebolledo, D. (2012). Aplicabilidad de los criterios de rigor y éticos en la investigación cualitativa. Aquichan, 12(3), 263-274. https://www.redalyc.org/articulo.oa?id=74124948006

Nussbaum, M. (1995). Poetic Justice. The literary imagination and public life. Beacon Press.

Nussbaum, M. (2009). Frontiers of justice. CNIB.

Oraisón y Pérez (2006). Escuela y participación: el difícil camino de la construcción de la ciudadanía. Revista lberoamericana de Educación, 42, 15-29. https://rieoei.org/historico/documentos/rie42a01.pdf

Pindado, F. (2000): La participación ciudadana en la vida de las ciudades. Ediciones del Serbal.

Pugh y De'Ath (1989). Towards Partnership in the Early Years. National Children's Bureau.

Seng, N.L., Hanafi, Z. y Taslikhan, M. (2016). Influence of parental involvement on academic achievement. International Journal of Advanced Education and Research, 1(4), 1-4. http://www.alleducationjournal.com/download/21/1-2-27.pdf

Subirats, M. (2014). La LOMCE: Hacia una educación antidemocrática. Revista Interuniversitaria de Formación del Profesorado, 28(3), https://www.redalyc.org/articulo.oa?id=27433841004

Taylor, S. y Bodgan, R. (1986). Introducción a los métodos cualitativos de investigación. Paidós.

Valles, M.S. (1997). Técnicas cualitativas de investigación social: Reflexión metodológica y práctica profesional. Síntesis. 
Fecha de entrada: 19 febrero 2020

Fecha de revisión: 10 julio 2020

Fecha de aceptación: 08 septiembre 2020 